ESAIM: PROCEEDINGS, Juillet 2013, Vol. 40, p. 34-50

C. Bourdarias, S. Gerbi, Editors

\title{
MODELLING COMPRESSIBLE MULTIPHASE FLOWS
}

\author{
Frédéric Coquel ${ }^{1}$, Thierry Gallouët ${ }^{2}$, Philippe Helluy ${ }^{3}$, Jean-Marc \\ HÉrard ${ }^{4}$, Olivier Hurisse ${ }^{5}$ AND Nicolas SEguin ${ }^{6}$
}

\begin{abstract}
We give in this paper a short review of some recent achievements within the framework of multiphase flow modeling. We focus first on a class of compressible two-phase flow models, detailing closure laws and their main properties. Next we briefly summarize some attempts to model two-phase flows in a porous region, and also a class of compressible three-phase flow models. Some of the main difficulties arising in the numerical simulation of solutions of these complex and highly non-linear systems of PDEs are then discussed, and we eventually show some numerical results when tackling twophase flows with mass transfer.

Résumé. Quelques résultats concernant la modélisation des écoulements multiphasiques Nous présentons dans cet article quelques résultats récents concernant la modélisation et la simulation numérique des écoulements multiphasiques. Nous nous concentrons tout d'abord sur une classe de modèles diphasiques compressibles, en détaillant les lois de fermeture et les principales propriétés du sytème. Nous résumons ensuite brièvement les propositions de modélisation d'écoulements diphasiques en milieu poreux et d'écoulements triphasiques. Quelques difficultés apparaissant dans la simulation numérique de ces modèles sont présentées, et des résultats récents comportant un transfert de masse entre phases sont finalement décrits.
\end{abstract}

\section{INTRODUCTION}

Following the initial quest of Ishii [29], the correct modelling of two-phase flows is still a widely debated topic, especially when focusing on the two-fluid approach, which aims at distinguishing mean properties of both phases (see for instance $[3,4,7,13,15-18,20,30,32,36,37]$ ). When restricting to the statistical averaging formalism, standard tools may be used in order to derive meaningful models, in order to tackle unsteady and inhomogeneous two-phase flow predictions. The present

${ }^{1}$ CMAP, Ecole Polytechnique, UMR CNRS 7671, route de Saclay, 91128, Palaiseau cedex, France

2 LATP, Université Aix-Marseille, UMR CNRS 7353, 39 rue Joliot Curie, 13453 Marseille cedex, France

${ }^{3}$ IRMA, 7 rue Descartes, Université de Strasbourg, 67084, Strasbourg, France

${ }^{4}$ EDF, R\&D, MFEE, 6 quai Watier, 78400, Chatou, France

${ }^{5}$ EDF, R\&D, MFEE, 6 quai Watier, 78400, Chatou, France

${ }^{6}$ LJLL, Université Pierre et Marie Curie,UMR CNRS 7598, Paris, France 
paper focuses on these models, and provides some closure laws that comply with an entropy inequality. The latter one is of course useful in order to control smooth and also -possibly- discontinuous solutions. One objective here is to provide a framework that might handle liquid-vapour mixtures, when the vapour phase is dilute (this corresponds to what might happen in the primary coolant circuit of nuclear power plants away from standard conditions), or when the mean flow contains a much larger amount of vapour (this may occur in the upper part of steam generators, or more likely in some severe accident configurations following the boiling crisis). Another issue is whether this framework may handle even more complex situations such as those arising when tackling threephase flows or flows in a porous medium.

Two-fluids models require the computation of eight unknowns (statistical void fractions, mean densities, mean velocities and mean pressures). Partial differential equations may be derived for statistical void fractions, and partial mass, momentum and total energy within each phase; however, equations of state which provide the mean internal energy within each phase must be prescribed, and some closure laws are thus necessary. Actually, it is usually assumed that the averaged EOS are functions of first-order moments only (mean pressure and mean density), though this is rigorously valid for some specific instantaneous EOS only (such as perfect gas EOS for instance). This assumption will be kept in the present work. Even more, closure laws must be given in order to account for interfacial transfer terms. We refer the reader to the work of Kapila, Glimm and other co-workers for that difficult topic (see $[4,5,19,20,31,32]$ ), and we only detail our methodology herein. Of course, depending on the choice of closure laws for interfacial quantities, properties of the closed set of partial differential equations may vary considerably.

Hence, the present paper aims at providing a quick review of some achievements in the framework of multiphase flow modelling. Among all constraints that should be satisfied in order to predict numerically unsteady flows containing shock patterns, the following three immediately arise:

(1) Systems are expected to be hyperbolic for all physically admissible states;

(2) Smooth solutions should comply with a relevant entropy inequality;

(3) Unique jump conditions should be available in order to caracterize shock solutions.

We try to describe the basic methodology that has been considered for two-phase flow modelling of unsteady flows involving discontinuities, but we also focus in the following section on some possible extensions to the framework of flows in a porous medium and three-phase flow predictions. A short synthesis of the numerical schemes that have been developped will complete this first part. Next we will turn to some practical and recent computations, with special focus on difficulties arising when the mass transfer is accounted for.

\section{TWO-PHASE FLOW MODELLING}

Throughout the paper, $\alpha_{k}(x, t)$ will denote the statistical void fraction of phase $k=l, v$, and will comply with the constraint $\alpha_{l}(x, t)+\alpha_{v}(x, t)=1$. Variables $\rho_{k}, U_{k}, P_{k}$ respectively denote the mean density, the mean velocity, the mean pressure within phase $k$, and we define partial masses

$m_{k}=\alpha_{k} \rho_{k}$. The total energy $E_{k}$ within phase $k=l, v$ is defined by: $E_{k}=\rho_{k} e_{k}\left(P_{k}, \rho_{k}\right)+\rho_{k}\left(U_{k}^{2}\right) / 2$, where $e_{k}\left(P_{k}, \rho_{k}\right)$ stands for the internal energy. The state variable $W$ will be noted :

$$
W^{t}=\left(\alpha_{l}, \alpha_{v}, m_{l}, m_{v}, m_{l} U_{l}, m_{v} U_{v}, \alpha_{l} E_{l}, \alpha_{v} E_{v}\right)
$$


Thus, when neglecting the contribution of viscous effects and turbulence, the form of the governing equations of mean quantities in the two-fluid model is:

$$
\begin{aligned}
& \partial_{t}\left(\alpha_{k}\right)+V_{i n t}(W) \partial_{x}\left(\alpha_{k}\right)=\phi_{k}(W) \\
& \partial_{t}\left(\alpha_{k} \rho_{k}\right)+\partial_{x}\left(\alpha_{k} \rho_{k} U_{k}\right)=\Gamma_{k}(W) \\
& \partial_{t}\left(\alpha_{k} \rho_{k} U_{k}\right)+\partial_{x}\left(\alpha_{k} \rho_{k} U_{k}^{2}\right)+\partial_{x}\left(\alpha_{k} P_{k}\right)-\Pi_{i n t}(W) \partial_{x}\left(\alpha_{k}\right)=D_{k}(W)+\Gamma_{k}(W) \bar{U}_{i n t} \\
& \partial_{t}\left(\alpha_{k} E_{k}\right)+\partial_{x}\left(\alpha_{k} U_{k}\left(E_{k}+P_{k}\right)\right)+P_{\text {int }}(W) \partial_{t}\left(\alpha_{k}\right)=\psi_{k}(W)+\bar{U}_{i n t} D_{k}(W)+\Gamma_{k}(W) \bar{H}_{i n t}
\end{aligned}
$$

Contributions $\Gamma_{k}(W), D_{k}(W)$ and $\psi_{k}(W)$ take interfacial mass transfer, drag effects and interfacial heat transfer into account. Besides, the term $\phi_{k}(W)$ arising in the governing equation of the statistical void fraction $\alpha_{k}$ is due to the statistical averaging [25] of the topological equation [13]. Obviously, we must enforce the following:

$$
\sum_{k=l, v} \Gamma_{k}(W)=0 \quad ; \quad \sum_{k=l, v} \psi_{k}(W)=0 \quad ; \quad \sum_{k=l, v} D_{k}(W)=0 \quad ; \quad \sum_{k=l, v} \phi_{k}(W)=0 .
$$

Interfacial terms

$$
\bar{U}_{\text {int }}=\left(U_{l}+U_{v}\right) / 2 \quad, \quad \bar{H}_{\text {int }}=U_{l} U_{v} / 2
$$

enable to account for mass and momentum transfer terms in the governing equations of mean velocities and mean total energies. Our main objective here is to determine some admissible form of all unknown quantities $\Gamma_{l}(W), \phi_{l}(W), \psi_{l}(W), D_{l}(W)$ and $\Pi_{\text {int }}(W), P_{\text {int }}(W)$, assuming some given convex combination for $V_{i n t}(W)$ in terms of $U_{l}, U_{v}$ :

$$
V_{\text {int }}(W)=\xi(W) U_{l}+(1-\xi(W)) U_{v} .
$$

where $\xi(W)$ lies in $[0,1]$. Physically relevant functions $\xi(W)$ have been proposed in $[7,16]$, and these will be recalled at the end of this section. We also denote $c_{k}$ the speed of acoustic waves within the pure $k$-phase, setting:

$$
\rho_{k} c_{k}^{2}=\left(\partial _ { P _ { k } } ( e _ { k } ( P _ { k } , \rho _ { k } ) ) ^ { - 1 } \left(\frac{P_{k}}{\rho_{k}}-\rho_{k} \partial_{\rho_{k}}\left(e_{k}\left(P_{k}, \rho_{k}\right)\right)\right.\right.
$$

\subsection{Entropy inequality}

We introduce the specific entropy $S_{k}\left(P_{k}, \rho_{k}\right)$ in each phase, which complies with:

$$
c_{k}^{2} \partial_{P_{k}}\left(S_{k}\right)+\partial_{\rho_{k}}\left(S_{k}\right)=0
$$

and temperatures: $1 / T_{k}=\partial_{P_{k}}\left(S_{k}\right) / \partial_{P_{k}}\left(e_{k}\right)$. We also set: $\mu_{k}=e_{k}+P_{k} / \rho_{k}-T_{k} S_{k}$, which is the Gibbs potential, classically associated with the description of phase transition. We may first assume that the following constraint holds:

$$
\Pi_{i n t}(W)-P_{\text {int }}(W)=0
$$

and also that $\Pi_{\text {int }}(W)$ is a convex combination of both pressures, that is:

$$
\Pi_{i n t}(W)=\chi(W) P_{l}+(1-\chi(W)) P_{v}
$$




\section{Proposition 1:}

We define:

$$
\chi(W)=(1-\xi(W)) / T_{l}\left[(1-\xi(W)) / T_{l}+\xi(W) / T_{v}\right]^{-1}
$$

If $W$ denotes a smooth solution of (1), the governing equation of the entropy of the two-fluid model $\eta(W)=\sum_{k=l, v} m_{k} S_{k}$ may be written as follows:

$$
\begin{aligned}
& \partial_{t}(\eta(W))+\partial_{x}\left(\sum_{k=l, v} m_{k} U_{k} S_{k}\right)=\quad \Gamma_{l}(W)\left(\mu_{v}(W) / T_{v}-\mu_{l}(W) / T_{l}\right) \\
& +\quad D_{l}(W)\left(U_{v}-U_{l}\right)\left(1 /\left(2 T_{v}\right)+1 /\left(2 T_{l}\right)\right) \\
& +\quad \psi_{l}(W)\left(T_{v}-T_{l}\right) /\left(T_{v} T_{l}\right) \\
& +\quad \phi_{l}(W)\left(P_{l}-P_{v}\right)\left(1 /\left(2 T_{v}\right)+1 /\left(2 T_{l}\right)\right)
\end{aligned}
$$

The proof requires rather long calculations and is thus omitted. Obviously, when $\xi(W)=0$ (or $\xi(W)=1)$, one retrieves the standard Baer-Nunziato model, where the interface velocity $V_{\text {int }}(W)$ corresponds to the mean velocity of the vanishing phase (see [3,4,32] and [17] also). This entropy budget has a straightforward counterpart in the three-dimensional framework.

- Since all quantities: $T_{v}-T_{l}, U_{v}-U_{l}, P_{v}-P_{l}, \mu_{v} / T_{v}-\mu_{l} / T_{l}$ are independent quantities, the following admissible closure laws arise:

$$
\begin{aligned}
& \Gamma_{l}(W)=K_{\Gamma}(W)\left(\mu_{v}(W) / T_{v}-\mu_{l}(W) / T_{l}\right) \\
& D_{l}(W)=K_{U}(W)\left(U_{v}-U_{l}\right) \\
& \psi_{l}(W)=K_{T}(W)\left(T_{v}-T_{l}\right) \\
& \phi_{l}(W)=K_{P}(W)\left(P_{l}-P_{v}\right)
\end{aligned}
$$

The first three closure laws were expected, and the last one is physically relevant: it simply means that the statistical void fraction of the liquid phase increases when the statistical pressures are such that: $P_{l}>P_{v}$. The -positive- scalar functions in the drag contribution and in the heat transfer closure law may be chosen as:

$$
\begin{gathered}
K_{U}(W)=m_{l} m_{v} /\left(m_{l}+m_{v}\right) / \tau_{U}(W), \\
K_{T}(W)=m_{l} m_{v} C_{l-v} /\left(m_{l}+m_{v}\right) / \tau_{T}(W),
\end{gathered}
$$

and hence agree with the classical two-fluid litterature [29]; moreover, a relevant choice for $K_{P}$ that preserves positive values of void fractions is:

$$
K_{P}(W)=\alpha_{l} \alpha_{v} /\left(P_{l}+P_{v}\right) /\left(\left|P_{l}\right|+\left|P_{v}\right|\right) / \tau_{P}(W)
$$

Here, $\tau_{U, P, T}(W)$ respectively denote velocity-pressure-temperature relaxation time scales. We also set: $K_{\Gamma}(W)=K_{\Gamma}^{\prime}(W) / \tau_{\Gamma}(W)$. The literature suggests physically sounded forms for $\tau_{U}(W)$ and $\tau_{T}(W)$ on the one hand; on the other hand, accurate time scales $\tau_{P}(W)$ can hardly be found in the available two-phase flow literature. Most of the time, it is more or less assumed to lie within the range $\left(\tau_{U}(W), \tau_{\Gamma}(W)\right)$. 
- The closure law (8) is exactly the one that has been introduced in $[7,16]$. Slightly different forms that account for the statistical void fraction gradient have been proposed later on [24], but they involve another scalar coefficient function that can hardly be determined experimentally, and are thus disregarded here. Other closure laws were proposed in [36]. Another point that is worth being emphasized is that it can be proved that the constraint (6) actually holds.

- The specific forms of $\bar{U}_{i n t}$ and $\bar{H}_{\text {int }}$ (see (3)) are those that guarantee that the relative velocity $U_{v}-U_{l}$ has no contribution in the entropy production function, when some mass transfer occurs between phases.

- All closure laws presented above may be used for a broader class of two-fluid models [25]. The main advantage of this latter class is that it may take transition regimes into account, which is useful for the prediction of the ebulition crisis or of water-hammer situations. The approach detailed here may also be used in order to tackle the modelling of three-phase flows [23]. This particular point will be discussed in the following section.

\subsection{Main properties}

We recall below the main properties of the homogeneous model associated with (1).

\section{Property 1:}

- The set of equations associated with the left-hand side of (1) has seven real eigenvalues which read:

$$
\begin{array}{rr}
\lambda_{1}=V_{\text {int }}(W) \\
\lambda_{2}=U_{v}, \quad \lambda_{3}=U_{v}-c_{v}(W), & \lambda_{4}=U_{v}+c_{v}(W), \\
\lambda_{5}=U_{l}, \quad \lambda_{6}=U_{l}-c_{l}(W), & \lambda_{7}=U_{l}+c_{l}(W)
\end{array}
$$

Associated righteigenvectors span the whole space $\mathcal{R}^{7}$, unless $\left|U_{k}-V_{\text {int }}(W)\right| / c_{k}=1$, for $k=l, v$;

- Fields associated with eigenvalues $\lambda_{2,5}$ are linearly degenerate. Other fields associated with eigenvalues $\lambda_{3,4,6,7}$ are genuinely nonlinear. The 1 -field is linearly degenerate if:

$$
\xi(W)(1-\xi(W))=0, \quad \text { or: } \quad \xi(W)=m_{l} /\left(m_{l}+m_{v}\right)
$$

- Smooth solutions of (1) comply with the entropy inequality:

$$
0 \leq \partial_{t}(\eta(W))+\partial_{x}\left(\sum_{k=l, v} m_{k} U_{k} S_{k}\right)
$$

when using closure laws (9) and (8).

- Unique jump conditions hold within each isolated field for discontinuous solutions of (1) when using closure laws (13).

This result is classical (see $[7,16]$ ). When the 1 -field is linearly degenerate, unique jump conditions can be written within each single field. Thus, we may expect that suitable schemes will provide a unique converged approximation when the mesh is refined, whatever the convective scheme is; this 
has been confirmed by numerous non-resonant numerical experiments $[11,18,22,27,34,38]$. This is indeed a remarkable property that has only raised little attention in the two-phase flow community. We emphasize that these particular choices for $\xi(W)$ detailed in (13) are totally independent from the specific choice (8); however both formulas (13) and (8) have been considered in our overall methodology. A straightforward consequence at this stage is that the homogeneous part of system (1) is a closed set of PDE.

\section{Some EXTENSIONS OF THE TWO-FLUID FORMALISM}

Among all possible extensions of the two-fluid formalism that has been described above, we would like to point out at least two different situations. The first one refers to the modelling of two-phase flows in a porous region; this point was basically motivated by the need for "component" codes in the nuclear safety framework, where the notion of porosity naturally arises when tiny obstacles in steam generators and cores are accounted for without meshing all boundaries. The second one is an innovative approach in an attempt to model three-field flows, by adopting a wider three-phase flow formalism. This is discussed in the following two subsections.

\subsection{A two-fluid model in a porous region}

When the physical domain is occupied by a fluid and rigid boundaries (including walls, solid obstacles, grids,...), a possible and widely used approach consists in defining the local porosity as the ratio of the volume of fluid $\mathcal{V}_{f}(\mathbf{x})$ over the total control volume $\mathcal{V}_{\text {total }}(\mathbf{x})=\mathcal{V}_{f}(\mathbf{x})+\mathcal{V}_{s}(\mathbf{x})$, where $\mathcal{V}_{s}(\mathbf{x})$ stands for the volume of solid, that is:

$$
\epsilon(\mathbf{x})=1-\mathcal{V}_{s}(\mathbf{x}) / \mathcal{V}_{\text {total }}(\mathbf{x})
$$

Thus, if one aims at adopting the two-fluid formalism, this leads to the problem of defining a meaningful set of PDEs, such as:

$$
\begin{aligned}
& \partial_{t}(\epsilon)=0 \\
& \partial_{t}\left(\alpha_{k}\right)+V_{i n t}(W) \partial_{x}\left(\alpha_{k}\right)=\phi_{k}(W) \\
& \partial_{t}\left(\epsilon m_{k}\right)+\partial_{x}\left(m_{k} U_{k}\right)=\epsilon \Gamma_{k}(W) \\
& \partial_{t}\left(\epsilon m_{k} U_{k}\right)+\partial_{x}\left(\epsilon m_{k} U_{k}^{2}\right)+\epsilon \alpha_{k} \partial_{x}\left(P_{k}\right)-\epsilon\left(\Pi_{i n t}(W)-P_{k}\right) \partial_{x}\left(\alpha_{k}\right)=\epsilon\left(D_{k}(W)+\Gamma_{k}(W) \bar{U}_{i n t}\right) \\
& \partial_{t}\left(\epsilon \alpha_{k} E_{k}\right)+\partial_{x}\left(\epsilon \alpha_{k} U_{k}\left(E_{k}+P_{k}\right)\right)+\epsilon \Pi_{i n t}(W) \partial_{t}\left(\alpha_{k}\right)=\epsilon\left(\psi_{k}(W)+\bar{U}_{i n t} D_{k}(W)+\Gamma_{k}(W) \bar{H}_{i n t}\right)
\end{aligned}
$$

for $k=l, v$. The latter system enjoys similar properties such as those detailed in section 2:

\section{Property 2:}

- The homogeneous part of system (15) is hyperbolic, unless some resonance occurs if $\mid U_{k}-$ $V_{\text {int }}(W) \mid / c_{k}=1$ or $\left|U_{k}\right| / c_{k}=1$; eigenvalues are:

$$
\begin{aligned}
& \lambda_{0}=0 \quad \lambda_{1}=V_{\text {int }}(W) \\
& \lambda_{2}=U_{v}, \quad \lambda_{3}=U_{v}-c_{v}(W), \quad \lambda_{4}=U_{v}+c_{v}(W), \\
& \lambda_{5}=U_{l}, \quad \lambda_{6}=U_{l}-c_{l}(W), \quad \lambda_{7}=U_{l}+c_{l}(W)
\end{aligned}
$$


- Smooth solutions of system (15) comply with the entropy inequality:

$$
0 \leq \partial_{t}(\epsilon \eta(W))+\partial_{x}\left(\epsilon \sum_{k=l, v} m_{k} U_{k} S_{k}\right)
$$

when closure laws are defined by (8) and (9).

- Fields associated with eigenvalues $\lambda_{0}=0, \lambda_{2}=U_{v}, \lambda_{5}=U_{l}$ are linearly degenerate, and a similar result holds for $\lambda_{1}=V_{\text {int }}(W)$ when the closure law $V_{i n t}(W)$ is chosen among (13).

These results are available in $[18,24]$. For practical applications in the nuclear framework, we emphasize that the resonance phenomenon is very unlikely to happen. Obviously, when sudden variations of $\epsilon$ arise in the computational domain, there is a missing term in the momentum equation, which means in practice that results obtained with (1) are more accurate than those coming from (15), unless some singular ad hoc source terms are added. Another point which is detailed in [18] is also worth being emphasized, which concerns the numerical approximation of solutions of system (15) when strong variations occur in the porous distribution $\epsilon(\mathbf{x})$. Actually, in that case, the computation of discontinuous solutions requires applying well-balanced schemes (with respect to the porous steady wave $\lambda=0$ ), otherwise approximations obtained with colocated Finite volume schemes may converge towards wrong solutions, even if these schemes involve approximate Riemann solvers. This implies that Riemann invariants of the steady wave should be perfectly preserved.

\subsection{A class of compressible three-phase flow models}

We focus now on a mixture of three phases, which will be indexed by $k=l, v, s$. We still use classical notations for the statistical fraction $\alpha_{k}$, the mean density, velocity and pressure $\rho_{k}, U_{k}, P_{k}$ within each phase $k$, and also denote the mass fractions $m_{k}=\alpha_{k} \rho_{k}$, and the total $k$-energy $E_{k}=\rho_{k} e_{k}\left(P_{k}, \rho_{k}\right)+\rho_{k}\left(U_{k}^{2}\right) / 2$. The three positive fractions $\alpha_{k}$ comply with:

$$
\Sigma_{k} \alpha_{k}=1
$$

The counterpart of the two-fluid compressible model (1) is now (see [23]):

$$
\begin{aligned}
& \partial_{t}\left(\alpha_{k}\right)+V_{\text {int }}(W) \partial_{x}\left(\alpha_{k}\right)=\phi_{k}^{t p f m}(W) \\
& \partial_{t}\left(m_{k}\right)+\partial_{x}\left(m_{k} U_{k}\right)=0 \\
& \partial_{t}\left(m_{k} U_{k}\right)+\partial_{x}\left(m_{k} U_{k}^{2}\right)+\partial_{x}\left(\alpha_{k} P_{k}\right)+\Sigma_{j \neq k} \Pi_{k, j}(W) \partial_{x}\left(\alpha_{j}\right)=D_{k}^{t p f m}(W) \\
& \partial_{t}\left(\alpha_{k} E_{k}\right)+\partial_{x}\left(\alpha_{k} U_{k}\left(E_{k}+P_{k}\right)\right)-\Sigma_{j \neq k} \Pi_{k, j}(W) \partial_{t}\left(\alpha_{j}\right)=V_{i}(W) D_{k}^{t p f m}(W)
\end{aligned}
$$

for $k$ and $j$ in $l, v, s$, when neglecting mass and energy interfacial transfer terms. Thus, closure laws must be provided not only for $\phi_{k}^{t p f m}(W)$ and $D_{k}^{t p f m}(W)$, but also for the six unknowns $\Pi_{k, l}(W)$, for $k \neq l$. A first series of constraints may be written:

$$
\begin{aligned}
& \Sigma_{k} \phi_{k}^{t p f m}(W)=0 \\
& \Sigma_{k} D_{k}^{t p f m}(W)=0 \\
& \Sigma_{k} \Sigma_{j \neq k} \Pi_{k, j}(W) \partial_{\chi}\left(\alpha_{l}\right)=0 \quad \text { for: } \quad \chi=x, t
\end{aligned}
$$

since these contributions account for interfacial transfer terms, and keeping in mind the fact that:

$$
\Sigma_{k} \partial_{x}\left(\alpha_{k}\right)=\partial_{x}\left(\Sigma_{k} \alpha_{k}\right)=0
$$


Moreover, it can be proved that the six unknowns $\Pi_{k, j}(W)$ uniquely depend on the other closure laws. Actually, introducing three functions $\beta_{k}(W)$, that represent the barycentric components of the sole velocity $V_{i n t}(W)$ in terms of the mean phasic velocities $U_{k}$, that is:

$$
V_{\text {int }}(W)=\beta_{l}(W) U_{l}+\beta_{v}(W) U_{v}+\beta_{s}(W) U_{s}
$$

and taking then into account the fact that the mixture entropy:

$$
\eta^{t p f m}=m_{l} S_{l}+m_{v} S_{v}+m_{s} S_{s}
$$

should be a mathematical entropy, we eventually get that the six functions $\Pi_{k, j}(W)$ can be written explictly in terms of the $\beta_{k}(W)$, the mean phasic pressures and temperatures $P_{k}, T_{k}$ (see [23], appendix G):

$$
\Pi_{k, j}(W)=\pi_{k, j}\left(\beta_{m}, P_{m}, T_{m}\right) \quad \text { with: } \quad m \in l, v, s .
$$

For instance, when choosing $\beta_{v}=1$ and $\beta_{l}=\beta_{s}=0$, we get at once:

$$
\Pi_{s, v}=\Pi_{v, s}=\Pi_{s, l}=P_{s} \quad ; \quad \Pi_{l, v}=\Pi_{v, l}=\Pi_{l, s}=P_{l}
$$

which turns to be the counterpart of the Baer-Nunziato formalism for three-phase flows.

This is in perfect agreement with the two-phase flow formalism discussed before. It also means that the methodology can be extended to a finite number of phases $N$ (though the algebra may become tedious when $N$ increases). Eventually, the only remaining unknowns are the interfacial transfer terms $\phi_{k}^{t p f m}(W)$ and $D_{k}^{t p f m}(W)$, but again, the entropy inequality:

$$
0 \leq \partial_{t}\left(\sum_{k=l, v, s} m_{k} S_{k}\right)+\partial_{x}\left(\sum_{k=l, v, s} m_{k} U_{k} S_{k}\right)
$$

provides a powerful tool in order to suggest suitable entropy-consistent closure laws for drag contributions between phases $D_{k}^{t p f m}(W)$, and for pressure relaxation terms $\phi_{k}^{t p f m}(W)$. We can summarize the main properties in the following:

\section{Property 3:}

- The homogeneous part of system (20) is hyperbolic, and eigenvalues are:

$$
\begin{aligned}
& \lambda_{1,2}=V_{\text {int }}(W), \\
& \lambda_{3}=U_{v}, \quad \lambda_{4}=U_{v}-c_{v}(W), \quad \lambda_{5}=U_{v}+c_{v}(W), \\
& \lambda_{6}=U_{l}, \quad \lambda_{7}=U_{l}-c_{l}(W), \quad \lambda_{8}=U_{l}+c_{l}(W), \\
& \lambda_{9}=U_{s}, \quad \lambda_{10}=U_{s}-c_{s}(W), \quad \lambda_{11}=U_{s}+c_{s}(W)
\end{aligned}
$$

Resonance may occur if $\left|U_{k}-V_{i n t}(W)\right| / c_{k}=1$, for $k=l, v, s$.

- Smooth solutions of system (20) comply with the entropy inequality:

$$
0 \leq \partial_{t}(\eta(W))+\partial_{x}\left(\sum_{k=l, v, s} m_{k} U_{k} S_{k}\right)
$$


when applying closure laws are defined by (23) for the $\Pi_{k, j}$ and also using admissible closure laws for interfacial transfer terms $D_{k}^{t p f m}(W), \phi_{k}^{t p f m}(W)$.

- Fields associated with eigenvalues $\lambda_{3}=U_{v}, \lambda_{6}=U_{l}, \lambda_{9}=U_{s}$ are linearly degenerate. If we note $M=m_{s}+m_{v}+m_{l}$, and if we assume that either $\left(\beta_{v}, \beta_{l}, \beta_{s}\right)=(1,0,0)$, or: $\left(\beta_{v}, \beta_{l}, \beta_{s}\right)=(0,1,0)$, or: $\left(\beta_{v}, \beta_{l}, \beta_{s}\right)=(0,0,1)$, or: $\left(\beta_{v}, \beta_{l}, \beta_{s}\right)=\left(m_{v} / M, m_{l} / M, m_{s} / M\right)$, then the field associated with $\lambda_{1,2}=V_{\text {int }}(W)$ is also linearly degenerate. In that case, unique jump conditions hold field by field for solutions of system (20). The latter conditions are sufficient but not necessary (the counterpart of the proposal [25] for three-phase flows might be considered).

The reader is refered to [23] for more details.

\section{Well-Suited Finite Volume SChemes}

We come back to two-phase flow modelling in a non-porous region, and focus on simple Finite Volume schemes that have been used for practical applications. Actually, the basic algorithm that is used to compute approximations of the whole system relies on an entropy-consistent fractional step method including an evolution step and a relaxation step. The evolution step computes approximate solutions of the pure convective system, and the relaxation step takes all source terms into account.

\subsection{Evolution step}

This step computes approximate solutions of the hyperbolic homogeneous system:

$$
\left\{\begin{array}{l}
\partial_{t}\left(\alpha_{k}\right)+V_{i n t}(W) \partial_{x}\left(\alpha_{k}\right)=0 \\
\partial_{t}\left(m_{k}\right)+\partial_{x}\left(m_{k} U_{k}\right)=0 \\
\partial_{t}\left(m_{k} U_{k}\right)+\partial_{x}\left(m_{k} U_{k}^{2}\right)+\partial_{x}\left(\alpha_{k} P_{k}\right)-\Pi_{i n t}(W) \partial_{x}\left(\alpha_{k}\right)=0 \\
\partial_{t}\left(\alpha_{k} E_{k}\right)+\partial_{x}\left(\alpha_{k} U_{k}\left(E_{k}+P_{k}\right)\right)+\Pi_{i n t}(W) \partial_{t}\left(\alpha_{k}\right)=0
\end{array}\right.
$$

through the time interval $\left[t^{n}, t^{n}+\Delta t\right]$, with given initial values $W^{n}$. The Finite Volume solver that is used in the last section can rely on a non-conservative version of the Rusanov scheme; in that case it ensures that partial masses remain positive, and also that statistical void fractions stay in the range $[0,1]$, provided that a specific CFL condition is enforced (see [18]). Actually, more accurate schemes such as the exact Riemann solver (see [39]), VFRoe-ncv approximate Riemann solver (see [16]), relaxation schemes (see $[1,2,6,10,38]$ ) or other schemes (see $[8,12,33,35,40]$ ) may be applied instead of the rough Rusanov scheme.

This evolution step provides a set of approximations $\tilde{W}$ at the end of each time step. 


\subsection{Relaxation step}

Given discrete cell values of $\tilde{W}$ at the beginning of each time step, we compute approximations of the coupled set of ODEs corresponding to relaxation terms, that is:

$$
\left\{\begin{array}{l}
\partial_{t}\left(\alpha_{k}\right)=\phi_{k}(W) \\
\partial_{t}\left(\alpha_{k} \rho_{k}\right)=\Gamma_{k}(W) \\
\partial_{t}\left(\alpha_{k} \rho_{k} U_{k}\right)=D_{k}(W)+\Gamma_{k}(W) \bar{U}_{i n t} \\
\partial_{t}\left(\alpha_{k} E_{k}\right)+\Pi_{i n t}(W) \partial_{t}\left(\alpha_{k}\right)=\psi_{k}(W)+\bar{U}_{i n t} D_{k}(W)+\Gamma_{k}(W) \bar{H}_{i n t}
\end{array}\right.
$$

Up to now, for a seek of simplicity and computational efficiency, all source terms have been decoupled, which means that other fractional steps are included, thus solving successively four separate steps:

$$
\left\{\begin{array}{l}
\partial_{t}\left(\alpha_{k}\right)=\delta_{\phi} \phi_{k}(W) \\
\partial_{t}\left(\alpha_{k} \rho_{k}\right)=\delta_{\Gamma} \Gamma_{k}(W) \\
\partial_{t}\left(\alpha_{k} \rho_{k} U_{k}\right)=\delta_{D} D_{k}(W)+\delta_{\Gamma} \Gamma_{k}(W) \bar{U}_{i n t} \\
\partial_{t}\left(\alpha_{k} E_{k}\right)+\Pi_{i n t}(W) \partial_{t}\left(\alpha_{k}\right)=\delta_{\psi} \psi_{k}(W)+\delta_{D} \bar{U}_{i n t} D_{k}(W)+\delta_{\Gamma} \Gamma_{k}(W) \bar{H}_{i n t}
\end{array}\right.
$$

associated with $\left(\delta_{\phi}, \delta_{\Gamma}, \delta_{D}, \delta_{\psi}\right)$ equal to $(1,0,0,0),(0,1,0,0),(0,0,1,0)$ and $(0,0,0,1)$ respectively.

The most difficult task in the building of the Finite Volume solver is due to the mass transfer term and to the contribution $\phi_{k}$. In particular, difficulties arise when enforcing the conservative form for the mixture, and meanwhile requesting void fractions in the physical range $[0,1]$ and positive densities and internal energies. Details on this part of the algorithm can be found in [26-28], and we only sketch the basic ideas below.

\subsubsection{Pressure relaxation step}

The pressure relaxation step computes approximations of solutions of:

$$
\left\{\begin{array}{l}
\partial_{t}\left(\alpha_{k}\right)=\phi_{k}(W) \\
\partial_{t}\left(\alpha_{k} \rho_{k}\right)=\partial_{t}\left(\alpha_{k} \rho_{k} U_{k}\right)=0 \\
\partial_{t}\left(\alpha_{k} E_{k}\right)+\Pi_{i n t}(W) \partial_{t}\left(\alpha_{k}\right)=0
\end{array}\right.
$$

A "fully implicit" discretization is applied (see $[15,26,27]$ ), which means that three main unknowns $\alpha_{v}^{*}, P_{v}^{*}, P_{l}^{*}$ are sought, that are solutions of:

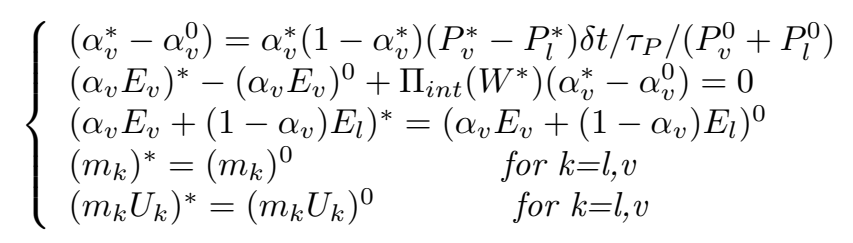

The problem of existence and uniqueness of the solution $\alpha_{v}^{*}, P_{v}^{*}, P_{l}^{*}$ in the physical range is examined in the latter references $[15,26,27]$. Obviously, the kind of EOS that is used within each phase is crucial, and the asymmetry of the BN model renders the investigation even more tricky. Another rather simple algorithm that does not preserve the conservative form of the total energy of the mixture was proposed earlier in [16] and is not recalled herein. 


\subsubsection{Gibbs potential relaxation step}

In order to take mass transfer into account, we need to obtain approximations of solutions of the following system:

$$
\left\{\begin{array}{l}
\partial_{t}\left(\alpha_{k}\right)=0 \\
\partial_{t}\left(m_{k}\right)=\Gamma_{k}(W) \\
\partial_{t}\left(m_{k} U_{k}\right)=\Gamma_{k}(W) \bar{U}_{i n t} \\
\partial_{t}\left(\alpha_{k} E_{k}\right)+\Pi_{i n t}(W) \partial_{t}\left(\alpha_{k}\right)=\Gamma_{k}(W) \bar{H}_{i n t}
\end{array}\right.
$$

A first simple scheme can be proposed in order to cope with these, that makes sense when the relaxation time step $\tau_{\Gamma}$ is not too small, and that preserves positive mass fractions (see [28]). However, a more convenient and general one is the following implicit scheme:

$$
\left\{\begin{array}{l}
m_{v}^{*}-m_{v}^{0}=\delta t \Gamma_{v}\left(W^{*}\right) \\
\left(m_{v} U_{v}\right)^{*}-\left(m_{v} U_{v}\right)^{0}=\delta t \Gamma_{v}\left(W^{*}\right) \bar{U}_{i n t}\left(W^{*}\right) \\
\left(\alpha_{v} E_{v}\right)^{*}-\left(\alpha_{v} E_{v}\right)^{0}=\delta t \Gamma_{v}\left(W^{*}\right) \bar{H}_{i n t}\left(W^{*}\right) \\
\alpha_{v}^{*}=\alpha_{v}^{0} \\
\left(m_{v}+m_{l}\right)^{*}=\left(m_{v}+m_{l}\right)^{0} \\
\left(\alpha_{v} E_{v}+\left(1-\alpha_{v}\right) E_{l}\right)^{*}=\left(\alpha_{v} E_{v}+\left(1-\alpha_{v}\right) E_{l}\right)^{0} \\
\left(m_{l} U_{l}+m_{v} U_{v}\right)^{*}=\left(m_{l} U_{l}+m_{v} U_{v}\right)^{0}
\end{array}\right.
$$

One drawback of course is that it requires solving a highly non-linear scalar equation (with respect to the unknown $m_{v}^{*}$ ), even when pure phasic equations of state are rough (such as perfect gas or stiffened gas EOS).

\section{A FEW RECENT COMPUtATional RESUlts}

We start with the verification of algorithms involved in the evolution step, and then show two different two-dimensional simulations with and without mass transfer.

\subsection{One-dimensional verification results based on Riemann problems}

The purpose of this subsection is to check the validity of the scheme that is used in the evolution step to predict convective effects. We consider the two-phase flow model (1), setting $V_{i n t}=U_{v}$ and $\Pi_{\text {int }}=P_{l}$, and we set formally:

$$
1 / \tau_{\Gamma}(W)=1 / \tau_{P}(W)=1 / \tau_{T}(W)=1 / \tau_{U}(W)=0
$$

Many Riemann problems have been considered in the verification of the evolution step, focusing either on first-order or second-order schemes. Exact solutions are computed by enforcing initial values for the left state $W_{L}$, and then computing the initial right state $W_{R}=\psi\left(W_{L}\right)$, where the transformation $\psi$ enables to account for all waves occuring in the solution.

We consider here a classical 1D Riemann problem that contains only two waves: a shock wave associated with the vapour phase, and a void fraction wave associated with $V_{i n t}=U_{v}$. The EOS are assumed to be perfect gas EOS within each phase $\left(\gamma_{v}=7 / 5\right.$ and $\left.\gamma_{l}=1.01\right)$. As it may be checked on figure 1, which plots the $L^{1}$ norm of the error with respect to the mesh size, while considering the rough first-order Rusanov scheme, the asymptotic rate of convergence is $h^{1 / 2}$, as it can be expected. The finer (resp. coarser) mesh contains 500000 (resp. 50) regular cells, and the CFL 


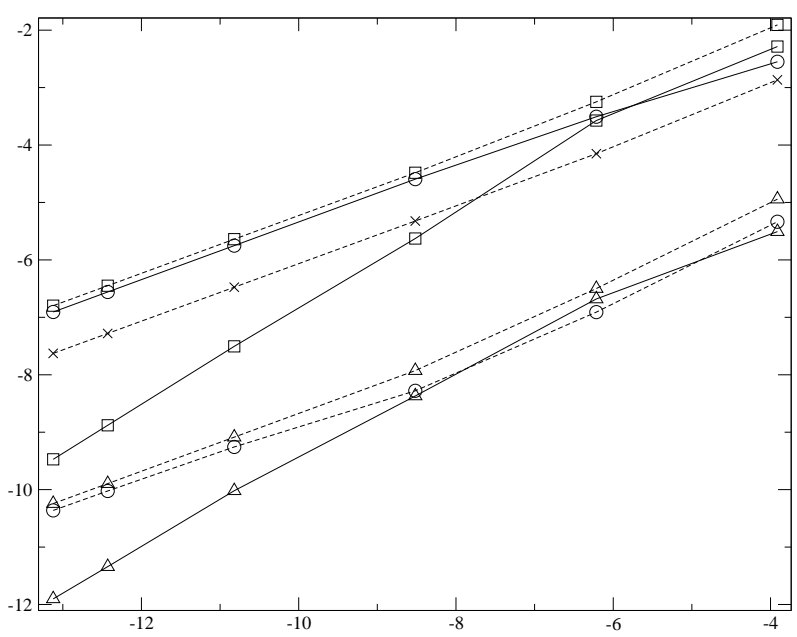

FiguRE 1. First-order Rusanov scheme : logarithm of the $L^{1}$ norm of the error $\left\|a-a_{h}\right\| /\|a\|$ as a function of $\log (h)$ for a 1D Riemann problem associated with step (30), for $a=\alpha, P_{v}, P_{l}, U_{v}, U_{l}, \rho_{v}, \rho_{l}$.

constraint is $C F L=1 / 2$ when computing approximate solutions of the convective system. The detailed analysis in [10] clearly shows that the efficiency of the relaxation solver proposed in the latter reference is much higher, compared with the rough Rusanov scheme, both in terms of accuracy and CPU time for a given level of accuracy. A thorough analysis of several Riemann problems is also available in $[10,11]$, where authors focus on first-order and second-order schemes. The extension to second-order was achieved using a classical minmod reconstruction based on symmetrizing variables $\left(\alpha_{k}, P_{k}, U_{k}, S_{k}\right)$ (see [9]) and a second-order Runge-Kutta time scheme. For second-order schemes, we retrieve the expected asymptotic rate of convergence $h^{2 / 3}$.

\subsection{Two-dimensional results without mass transfer}

We consider now the two-dimensional unsteady computation of a heated wall in which a small cavity has been inserted. The model is again based on (1), while setting : $V_{\text {int }}=U_{v}$ and $\Pi_{\text {int }}=P_{l}$. Relaxation time scales are now:

$$
\tau_{P}(W)=10^{-6}, \quad \tau_{U}(W)=10^{-4}, \quad \tau_{T}(W)=10^{-6}
$$

and $1 / \tau_{\Gamma}(W)=0$. We still use the first-order Rusanov scheme for practical computations. The computational domain contains $10^{5}$ regular cells, and the CFL number is again set to $1 / 2$. Homogeneous Neumann-type boundary conditions have been used on the left, right and upper boundaries. Uniform initial conditions are such that the fluid is at rest and in equilibrium at the beginning of the computation, and a uniform normal heat flux is imposed on the lower wall boundary. Figure 2 shows the liquid pressure profiles at the end of the computation. Actually, these 2D results still depend on the mesh refinement (though its counterpart in a 3D framework would contain more than 
30 millions cells). This confirms that current efforts in order to derive accurate Riemann solvers, or sufficiently large time-step schemes, are indeed mandatory.

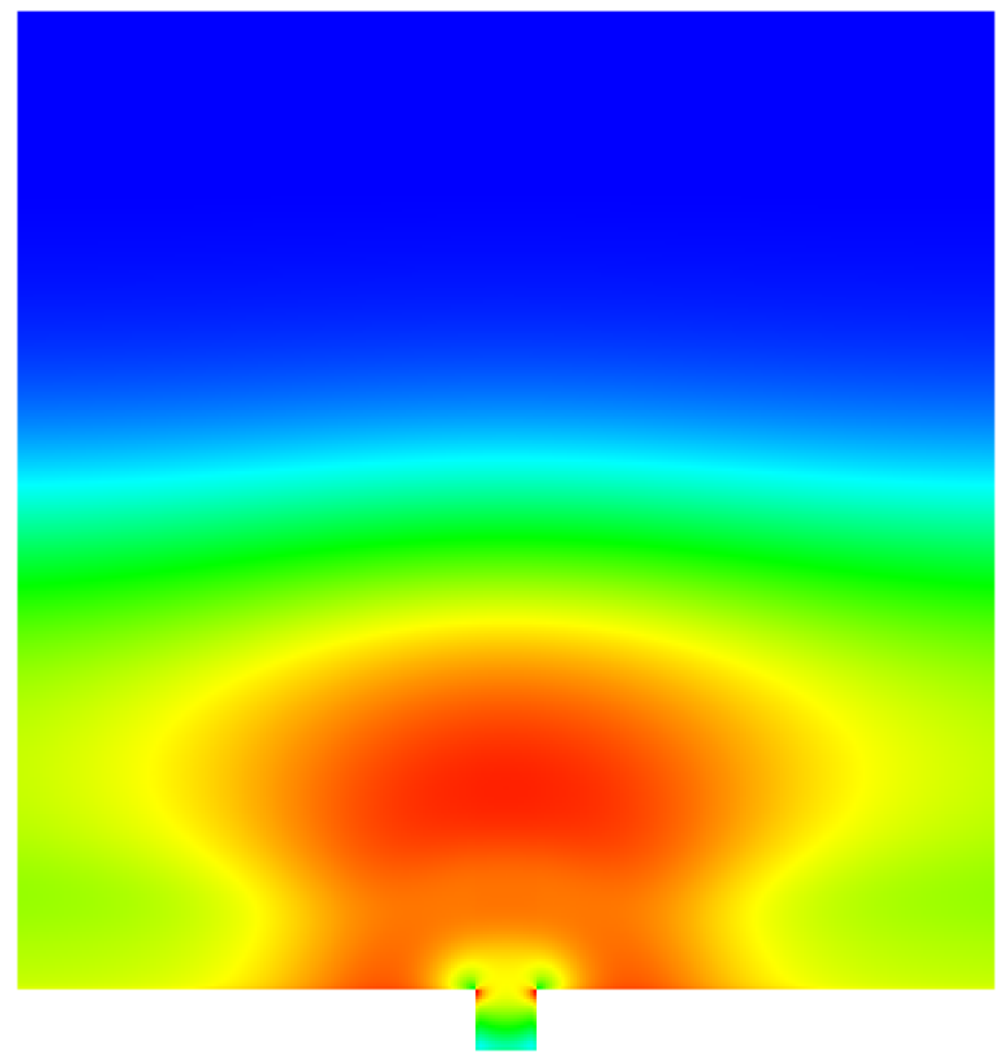

pression_L

$1.764 \mathrm{e}+07$

$1.739 \mathrm{e}+07$

$1.714 \mathrm{e}+07$

$1.688 \mathrm{e}+07$

$1.663 \mathrm{e}+07$

Figure 2. Heated wall: contours of the liquid pressure $P_{l}$ at time $T=0.0237$.

\subsection{Three-dimensional results including mass transfer}

The last test case is a preliminary result involving mass transfer between phases. We use now the relaxation scheme introduced in [38]. The implicit relaxation scheme (36) has been used in order to obtain numerical approximations. The system that is considered is still the same, though relaxation time scales are now the following:

$$
\tau_{P}(W)=10^{-4}, \quad \tau_{\Gamma}(W)=10^{-4}, \quad \tau_{U}(W)=10^{-4}, \quad \tau_{T}(W)=10^{-4} .
$$

The computational domain $\Omega=[0,7.4]$ corresponds to a long uniform pipe with a uniform heating along the wall boundary in the region $[0.7,5.9]$, the heat flux being equal to $H F=14 M W . x=0$ corresponds to the inlet boundary while $x=7.4$ is the outlet boundary. A perfect gas EOS is used for the vapour phase (with $\gamma_{v}=1.2462$ ), and a stiffened gas EOS is used for the vapour phase (with $\left.\gamma_{l}=1.6347, \Pi_{l}^{\infty}=5.207 \times 10^{8}\right)$. 
Initial conditions are also uniform in the pipe and have been set to the left inlet boundary conditions, which are:

$U_{v}^{\text {inlet }}=U_{l}^{\text {inlet }}=1.8281 \quad P_{v}^{\text {inlet }}=P_{l}^{\text {inlet }}=55.715 \times 10^{5} \quad \rho_{v}^{\text {inlet }}=28.053 \quad \rho_{l}^{\text {inlet }}=769.43$

while setting $\alpha_{l}^{\text {inlet }}=0.995$. Mean densities at the inlet are such that $T_{v}^{\text {inlet }}=T_{l}^{\text {inlet }}$. Both velocity components $V_{l, v}^{\text {inlet }}$ and $W_{l, v}^{\text {inlet }}$ have been set to zero. Two different meshes have been used, including 60 and 500 uniform cells along the axis. We have set $C F L=0.45$, thus time steps are approximately $\delta t=4 \cdot 10^{-5}$ and $\delta t=5 \cdot 10^{-6}$ for the coarse and the fine mesh respectively. Results are displayed at time $T=1$ on figures 3 and 4 . Both velocities $U_{l}$ and $U_{v}$ are increasing in the
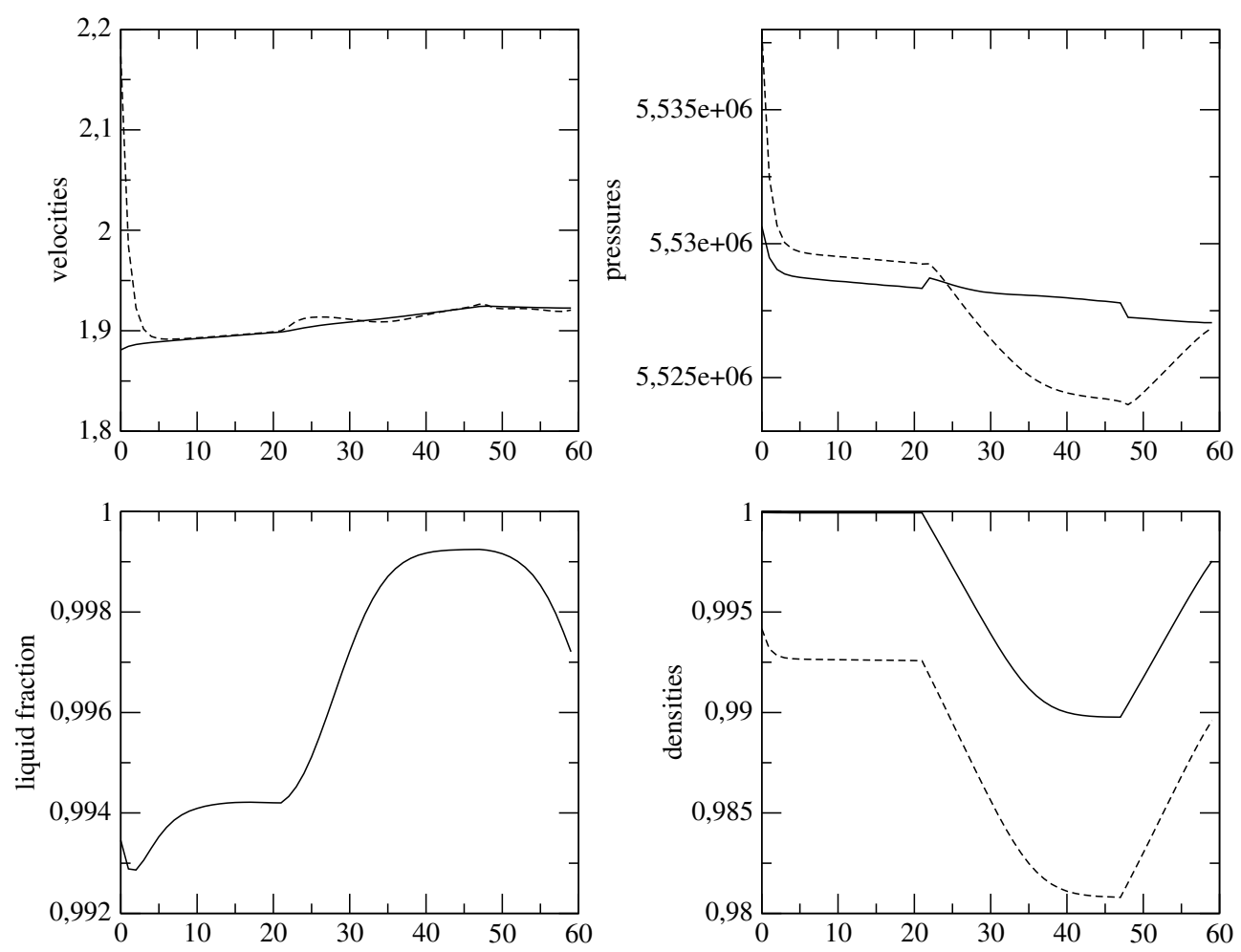

Figure 3. Heated pipe. Pressures, velocities, normalized densities $\rho_{k}(x, T) / \rho_{k}(0, T)$ and liquid fraction at time $T=1$. The plain line refers to the liquid phase, whereas the dashed line represents the vapour phase. The coarse mesh contains 60 regular cells.

heated region, while densities are decreasing in the same interval. The competition of the relaxation time scales results in an increase of the liquid fraction.

\section{Conclusion}

All multiphase flow models that have been described herein rely on the same methodology, and associated schemes are expected to provide meaningful convergent approximations of solutions of 

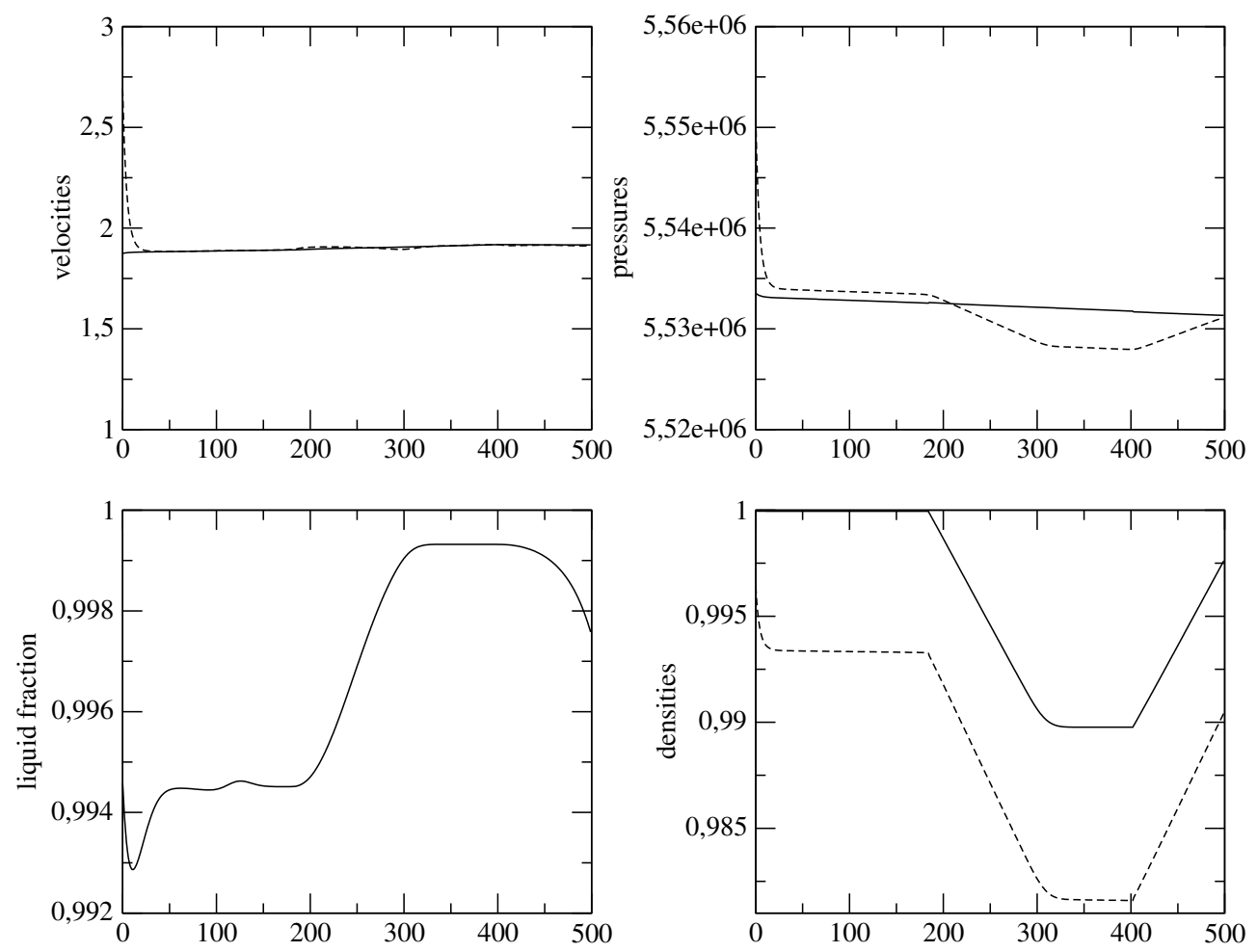

Figure 4. Heated pipe. Pressures, velocities, normalized densities $\rho_{k}(x, T) / \rho_{k}(0, T)$ and liquid fraction at time $T=1$. The plain line refers to the liquid phase, whereas the dashed line represents the vapour phase. The fine mesh contains 500 regular cells.

systems of PDEs. Basically, four distinct requirements sustain the whole approach, which are summarized below:

- The convective part of these systems is hyperbolic unless some resonance occurs in the solution;

- Smooth solutions are in agreement with a physically meaningful entropy inequality;

- Though non-conservative first-order contributions are present in these systems, unique jump conditions can be obtained;

- Finite Volume schemes are such that numerical approximations of Riemann problems converge towards unique solutions even when shocks are present.

According to the authors, this is an important improvement that should help in the assessment of multiphase flow models. However, it seems mandatory to point out the following drawbacks and main challenges.

First of all, there is a lack of physical knowledge about the four relaxation time scales $\tau_{U}, \tau_{P}, \tau_{T}, \tau_{\Gamma}$. In particular, little is known about the pressure relaxation time scale, since, up to now, most of the experimental effort has been put on the improvement of drag prediction and mass transfer. The 
correct estimation of $\tau_{P}$ thus requires further investigation and suitable experimental setups.

A second point is that these highly non-linear systems involve many different time scales that lie within a very wide range; a straightforward consequence is that high-order efficient and stable enough schemes are mandatory if one expects to get unsteady approximations that are not too far from mesh convergence. This urges the development of hybrid implicit-explicit schemes in order to obtain accurate approximations of components associated with slow internal waves. Relaxation schemes seem to provide a fair framework that might handle complex equations of state, and meanwhile provide accurate enough approximations.

Another difficulty corresponds to the simulation of transitional situations such as those that may be encountered in the prediction of flows in pressurised water rectors in severe accident configurations.

We would like to thank PhD students Laetitia Girault, Vincent Guillemaud, Yujie Liu, Khaled Saleh for their contributions. Part of the work of Jean-Marc Hérard and Olivier Hurisse has been achieved within the framework of the NEPTUNE project, which receives financial support by CEA, EDF, AREVA and IRSN. Nicolas Seguin has been supported by LRC Manon (Modélisation et approximation numérique orientées pour l'énergie nucléaire - CEA/DM2S-LJLL).

\section{REFERENCES}

[1] A. Ambroso, C. Chalons, F. Coquel, T. Galié, Relaxation and numerical approximation of a two-fluid twopressure diphasic model. ESAIM: M2AN, 43 (6), pp. 1063-1097 (2009).

[2] A. Ambroso, C. Chalons, P.A. Raviart, A Godunov-type method for the seven-equation model of compressible two-phase flow, Computers and Fluids, 54, pp67-91, 2012.

[3] M.R. Baer, J.W. Nunziato, A two-phase mixture theory for the deflagration to detonation transition (DDT) in reactive granular materials, IJMF, 12(6), pp. 861-889, (1986).

[4] J.B. Bdzil, R. Menikoff, S.F. Son, A.K. Kapila, D.S. Stewart, Two phase modelling of deflagration to detonation transition in granular materials: a critical examination of modelling issues, Phys. of Fluids, 11, pp. 378-402, (1999).

[5] W. Bo, H. Jin, D. Kim, X. Liu, H. Lee, N. Pestieau, Y. Yu, J. Glimm, J.W. Grove, Comparison and validation of multi phase closure models, Computers and mathematics with Applications, 56, pp. 1291-1302, (2008).

[6] C. Chalons, F. Coquel, S. Kokh, N. Spillane, Large time-step numerical scheme for the seven-equation model of compressible two-phase flows, in proceedings of Finite Volumes for Complex Applications VI, Prague, (2011).

[7] F. Coquel, T. Gallouët, J.M. Hérard, N. Seguin, Closure laws for a two-fluid two-pressure model, C. R. Acad. Sci. Paris, I-332, pp. 927-932, (2002).

[8] F. Coquel, J.M. Hérard, K. Saleh, A splitting method for the isentropic Baer-Nunziato two-phase flow model, ESAIM proceedings, 38, pp. 241-256 (2013).

[9] F. Coquel, J.M. Hérard, K. Saleh, N. Seguin, Two properties of two-velocity two-pressure models for two-phase flows, Communications in Mathematical Sciences, (2013).

[10] F. Coquel, J.M. Hérard, K. Saleh, N. Seguin, A robust entropy-satisfying riemann solver for the isentropic Baer-Nunziato model, submitted for publication.

[11] F. Crouzet, F. Daude, P. Galon, P. Helluy, J.M. Hérard, O. Hurisse, Y. Liu, Approximate solutions of the Baer-Nunziato model, submitted for publication, (2012).

[12] V. Deledicque, M. Papalexandris An exact Riemann solver for compressible two-phase flow models containing non-conservative products, J. Comp. Phys., 222, pp. 217-245, (2007).

[13] D.A. Drew, S.L. Passman, Theory of multi-component fluids, Applied Mathematical Sciences, 135, Springer, (1999).

[14] P. Embid, M. Baer, Mathematical analysis of a two-phase continuum mixture theory, Continuum Mech. Thermodyn., 4, pp. 279-312, (1992). 
[15] T. Gallouët, P. Helluy, J.M. Hérard, J. Nussbaum, Hyperbolic relaxation models for granular flows, ESAIM: M2AN, 44 (2), (2010).

[16] T. Gallouët, J.M. Hérard, N. Seguin, Numerical modelling of two phase flows using the two-fluid two-pressure approach, Math. Mod. Meth. in Appl. Sci., 14(5), pp. 663-700, (2004).

[17] S. Gavrilyuk, R. Saurel, Mathematical and numerical modelling of two-phase compressible flows with microinertia, J. Comp. Phys., 175, pp. 326-360, (2002).

[18] L. Girault, J.M. Hérard, A two-fluid hyperbolic model in a porous medium, ESAIM: M2AN, 44(6), pp. 13191348, (2010).

[19] J. Glimm, D. Saltz, D.H. Sharp, Renormalization group solution of two-phase flow equations for RayleighTaylor mixing, Phys. Letters A., 222, pp. 171-176, (1996).

[20] J. Glimm, D. Saltz, D.H. Sharp, Two-phase flow modelling of a fluid mixing layer, J. Fluid Mech., 378, pp. 119-143, (1999).

[21] S.K. Godunov, Finite difference method for numerical computation of discontinuous solutions of the equations of fluid dynamics, Mat. Sb., 47, pp. 271-300, (1959).

[22] V. Guillemaud, Modélisation et simulation des écoulements diphasiques par une approche bifluide à deux pressions, PhD thesis, Université Aix Marseille, Marseille, France, (2007).

[23] J.M. Hérard, A three-phase flow model, Math. and Computer Model., 45, pp. 732-755, (2007).

[24] J.M. Hérard, Un modèle hyperbolique diphasique bifluide en milieu poreux, Comptes-Rendus Mécanique, 336, pp. 650-655, (2008).

[25] J.M. Hérard, Une classe de modèles diphasiques bi-fluides avec changement de régime, internal EDF report H-I81-2010-0486-FR, in French, (2010).

[26] J.M. Hérard, O. Hurisse, Schémas d'intégration du terme de relaxation des pressions phasiques pour un modèle bifluide hyperbolique, EDF report H-I81-2009-01514-FR, (2009).

[27] J.-M. Hérard , O. Hurisse, A fractional step method to compute a class of compressible gas-liquid flows, Computers and Fluids, 55, pp. 57-69, (2012).

[28] J.-M. Hérard , O. Hurisse, Computing two-fluid models of compressible water-vapour flows with mass transfer, AIAA paper 2012-2959, http://www.aiaa.org/ (2012).

[29] M. Ishii, Thermofluid dynamic theory of two-phase flow, Collection de la Direction des Etudes et Recherches d'Electricité de France, Collection Eyrolles, (1975).

[30] M. Ishii, T. Hibiki, Thermofluid dynamics of two-phase flow, Springer, (2006).

[31] H. Jin, J. Glimm, D.H. Sharp, Compressible two-pressure two-phase flow models, Physics Letters, 353, pp. 469474, (2006).

[32] A.K. Kapila, S.F. Son, J.B. Bdzil, R. Menikoff, D.S. Stewart, Two phase modeling of a DDT: structure of the velocity relaxation zone, Phys. of Fluids, 9(12), pp. 3885-3897, (1997).

[33] S. Karni, G. Hernandez-Duenas, A hybrid algorithm for the Baer Nunziato model using the Riemann invariants, SIAM J. of Sci. Comput., 45, pp.382-403, (2010).

[34] Y. Liu , PhD thesis, Université Aix Marseille, Marseille, France, in preparation (2013).

[35] C.A. Lowe, Two-phase shock-tube problems and numerical methods of solution, J. Comp. Physics., 204, pp. 598-632, (2005).

[36] M. Papin, R. Abgrall, Fermetures entropiques pour les modèles bifluides à sept équations, Comptes-Rendus Mécanique, 333, pp. 838-842, (2005).

[37] V. Ransom, D.L. Hicks, Hyperbolic two-pressure models for two-phase flow, J. Comp. Physics., 53, pp. 124-151, (1984).

[38] K. Saleh, Analyse et simulation numérique par relaxation d'écoulements diphasiques compressibles. Contribution au traitement des phases évanescentes, PhD thesis, Université Pierre et Marie Curie, Paris, France, (2012).

[39] D.W. Schwendeman, C.W. Wahle, A.K. Kapila, The Riemann problem and a high-resolution Godunov method for a model of compressible two-phase flow, J. Comp. Physics., 212, pp. 490-526, (2006).

[40] S.A. Tokareva, E.F. Toro, HLLC type Riemann solver for the Baer-Nunziato equations of compressible twophase flow, J. Comp. Physics., 229, pp. 3573-3604, (2010). 